\title{
A Data Change Rule Based Empirical Framework for Labeling Unlabeled Time Series Driving Data
}

\author{
Supriya Sarker \\ Dept. of Computer Science \& Engineering \\ Chittagong University of Engineering and Technology \\ Chittagong, Bangladesh \\ sarkersupriya7@gmail.com
}

\author{
Md Mokammal Haque \\ Dept. of Computer Science \& Engineering \\ Chittagong University of Engineering and Technology \\ Chittagong, Bangladesh \\ mokammel@cuet.ac.bd
}

\begin{abstract}
Most of the driving maneuver classification methods follow supervised learning techniques and utilize ground truth in order to train classifiers. However, collecting ground truth is the most troublesome, expensive, and significant task of classification and effects a classifier's performance. The work proposes an empirical framework for automatic labeling of timeseries data that can be further used in training phrases during semi-supervised learning. The proposed algorithm generates class labels and find that generated label of $\mathbf{4 8 9 5}$ data matched with 11077 manual labeled data. The work analyzes the challenges involved in the driving time series data labeling. So, reasons behind mismatch of data label can also be explained.

Index Terms-driving maneuver classification, time series data, ground truth, class label, automatic labeling, data change rule, rule based labeling
\end{abstract}

\section{INTRODUCTION}

Technological advancements result in an immense impact on the automobile industry. With the increasing number of vehicles, road accident is one of the catastrophic incidents still now. To abate the loss caused by traffic mishaps, many researchers proposed and developed different solutions [1][4]. These solutions are a means of curtailing the loss after a mishap. For avoiding accidents and ensure traffic safety, traffic events need to be predicted before taking place. Different traffic events are intimately related to driving maneuver of drivers, that is why the driving patterns of drivers are one of the major reason of traffic mishap. Researchers have proposed different classification techniques to predict maneuvers that are prone to accidents in [5]- [8]. Among those techniques, the most common approach is time series classification techniques that are based on supervised learning. So the used dataset is needed to be labeled into different classes or categories to train the classifiers [9]. These ground truth data has a significant influence on the performance and accuracy of the classifier. Therefore, the accumulation of the ground truth is a very important but most arduous, time-consuming, and expensive task. To acquire the ground-truth label, during data collection the situation is recorded by video recording or manually by handwritten memo [10]. In [11] transport mode has been classified by identifying breakpoints of a signal by finding points in time when the signal's variance changes significantly during the transition from one mode to another. Cardoso et al. in [12] predicted confidence of the unlabeled dataset and included in the labeled dataset unless beyond a defined threshold of their classifier. In [9], He et al. measured the uncertainty of the unlabeled sample by calculating the similarity distance from a positive labeled sample for multivariate time series classification. Murao and Terada [10] partitioned unlabeled data into segments and clustered each of them to find the best match of patterns. However, the classification of time series sequence data using these techniques is not possible while the time-series dataset contains complex patterns and ambiguous classes. Besides, time series classification may vary for different domains and needed prior domain knowledge to deal with. Therefore, in this work, we combine the data changing rules of sensor fusion time-series driving data and rule based models with domain knowledge.

The main contribution made in this research is (i) develop a framework for labeling unlabeled time series driving data (ii) analyze the challenges involved in time series sensor fusion driving data labeling.

\section{Moving Vehicle Data Change Rules}

Supervised machine learning algorithms serve excellently by predicting classes of time series even in some unpredictable situations. However, the possible outcome of time series collected from real-life scenarios is ambiguous to an extent. Many researchers have ignored the domain knowledge of moving vehicles for classification of driving maneuvers using sensors data. During movement subject vehicle's sensor fusion data follows specific change rules which can be explained by the kinematics of a rigid body and discussed in [13] and as a result, bring significant and meaningful changes in time series patterns. The change of data pattern for a particular maneuver is depicted in Fig. 1 where $a_{x}, a_{y}$ and $w_{z}$ denote the time series of $x$ and $y$-axis of accelerometer, and $z$-axis of the gyroscope. During movement in the real scenarios, a vehicle experiences longitudinal, lateral, and angular velocity represented by $a_{x}$, $\mathrm{a}_{\mathrm{y}}$ and $\mathrm{w}_{\mathrm{z}}$, respectively. Moving straight causes a change in longitudinal i.e., $\mathrm{a}_{\mathrm{x}}$ data and lane change, turning cause shift of both $\mathrm{a}_{\mathrm{y}}$ and $\mathrm{w}_{\mathrm{z}}$ data. Based on the data change rules, Sarker and Haque in [14] proposed a few hypotheses which are proved by experiment with time series driving labeled dataset.

Those hypotheses provide some ranges of time series during a particular event. Fig. 1 implies that during acceleration and 


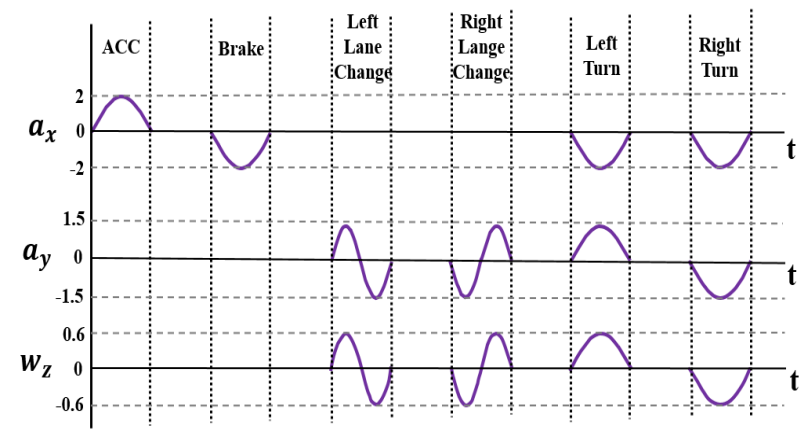

_ Time series data of sensors different axes during particular maneuver

Fig. 1. Demonstration of data changing rule of time series driving data

braking, time-series reaches from zero to 2 and -2 , respectively which is the non-aggressive range of $a_{x}$. For left lane change (LLC) and right lane change (RLC), it is not significant. During the left turn (LT) and right turn (RT), it follows the changing pattern of braking. During LT and RT, $\mathrm{a}_{\mathrm{y}}$ reaches almost 1.5 from zero and goes to the same amount in negative axis, respectively. For the same maneuvers, $\mathrm{w}_{\mathrm{z}}$ reaches 0.2 from zero and goes to -0.2 , respectively. It is notable that the pattern of LLC and RLC can be formed by combining LT-RT and RT-LT sequences (see Fig. 2) for both $a_{y}$ and $w_{z}$. As the time series patterns are very similar to each other, the classifier gets confused. For instance, RLC is classified as RT and vise-versa proved in classification with LSTM model in [14]. Therefore, we utilized a set of predefined rules to label unlabeled time-series driving dataset and guide the classifier model.

\section{Proposed Methodology}

\section{A. Preparation of Dataset}

Let Class $=\left\{\right.$ class $_{1}$, class $_{2}, \ldots$, class $\left._{n}\right\}$ be a set of driving maneuver classes and a single priority value is associated with class $_{i}$ denoted by Class prior $=\{1,2, \ldots, n\}$. TS is a collection of time-series dataset [15] collected by an accelerometer and gyroscope sensor denoted by $T S=\left\{t_{1}, t_{2}, \ldots, t_{n}\right\}$. For every $\mathrm{t}_{\mathrm{i}}$ there are three set of sensor data value $\left(\mathrm{a}_{\mathrm{x}_{\mathrm{i}}}, \mathrm{a}_{\mathrm{y}_{\mathrm{i}}}, \mathrm{w}_{\mathrm{z}_{\mathrm{i}}}\right)$. Class Rule, $R=\left\{r_{1}, r_{2}, \ldots, r_{n}\right\}$ be the set of rules applied on the sensor data $\left(\mathrm{a}_{\mathrm{x}_{\mathrm{i}}}, \mathrm{a}_{\mathrm{y}_{\mathrm{i}}}, \mathrm{w}_{\mathrm{z}_{\mathrm{i}}}\right)$ to label a discrete time-series $\mathrm{t}_{\mathrm{i}}$.

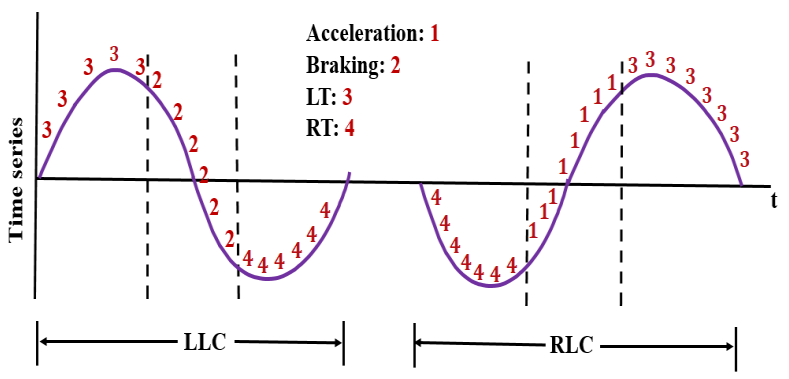

Fig. 2. Demonstration of pattern sequence of LLC and RLC combining LTBrake-RT and RT-ACC-LT

\section{B. Feature Set Extraction}

Among many statistical features, we deal with $\mathrm{a}_{\mathrm{x}_{\mathrm{i}}}, \mathrm{a}_{\mathrm{y}_{\mathrm{i}}}$ and $\mathrm{w}_{\mathrm{z}_{\mathrm{i}}}$ time-series data and slope, and energy of those to some extent because they provide the best performance. The notable change of the mentioned features during particular maneuver has been discussed in [14]. Initially, we drop the unlabeled time series and consider only the labeled dataset to generate the same ground truth by applying the proposed rule-based algorithm. By applying the algorithm, we find the new feature sequences for each driving maneuver class. Table I shows a small fragment of new feature space.

\section{Develop Class Rule Set}

We set non-aggressive specific threshold value for $a_{x_{\mathrm{i}}}, a_{y_{\mathrm{i}}}$ and $\mathrm{w}_{\mathrm{z}_{\mathrm{i}}}$ time series and develop $\mathrm{r}_{1}, \mathrm{r}_{2}, \mathrm{r}_{3}$ where we consider non-aggressive data range of times series and corresponding energy as propositions and combine them with propositional logic. $r_{4}$ and $r_{5}$ utilize $r_{1}$ with logical OR operation of an aggressive range of slope of acceleration and braking, respectively. As for every maneuver, the slope of time series lies both in the positive and negative axis which causes unnecessary confusion in decision making. On the other hand, the absolute value of slope indicates a difference between acceleration and braking, LT and RT. Hence, we consider the absolute value of the slope as the slope in the rule set. The significant ranges of time series data for a particular maneuver are illustrated in Fig. 3 and the Class Rule Set are listed in Table II.

New feature set for each class is created by applying the Algorithm. 1. From all the new feature set, the Algorithm. 3 selects the most first priority class priority value as discrete time series label value. The label value is mapped with the class name. As we see in Fig. 2 the combined feature of LTBrake-RT and RT-ACC-LT. We apply shift operation to update a sequence of class priority while there are seldom different priority values in a feature sequence. The shift and update process makes the result much better. But it is very difficult to make the decision on how much to shift because every event is uncertain and spontaneous in real driving situation. If we pay attention to Fig. 2, pattern matching is very hard while we do not know how long the LLC or RLC take place in a real driving situation. The longer the events exist the large the time series i.e., patterns will be. At the same time, it depends

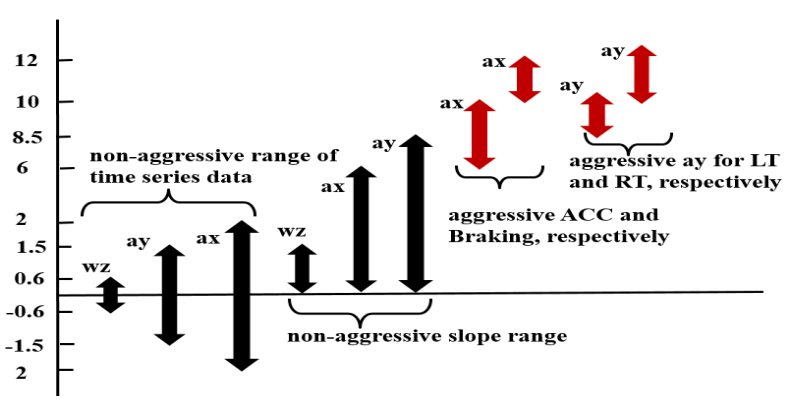

Fig. 3. Non-aggressive and Aggressive ranges of time series 
TABLE I

A fragment of Class Feature Set

\begin{tabular}{|c|c|c|c|c|c|c|c|c|}
\hline TS & agg & acc & $\begin{array}{c}\text { brak } \\
\text { ing }\end{array}$ & LT & RT & $\begin{array}{c}\text { comb } \\
\text { LT } \\
\text { _brak } \\
\mathbf{e} \_ \text {RT }\end{array}$ & $\begin{array}{c}\text { comb } \\
\text { RT } \\
\text {-acc } \\
\text { _LT }\end{array}$ & Label \\
\hline $\mathrm{t}_{1}$ & 0 & 1 & 2 & 0 & 0 & 1 & 1 & 0 \\
\hline $\mathrm{t}_{2}$ & 0 & 1 & 2 & 0 & 0 & 1 & 1 & 0 \\
\hline $\mathrm{t}_{3}$ & -1 & 1 & 2 & -1 & 4 & 4 & 1 & 1 \\
\hline$\ldots$ & $\ldots$ & $\ldots$ & $\ldots$ & $\ldots$ & $\ldots$ & $\ldots$ & $\ldots$ & $\ldots$ \\
\hline $\mathrm{t}_{\mathrm{n}}$ & -1 & 1 & -1 & 3 & 4 & 4 & 1 & 1 \\
\hline
\end{tabular}

on the data collection rate of sensors per unit time. Algorithm. 2 find such pattern.

\section{RESUlts AND ANALYSIS}

\section{A. Experimental Results}

In this section, we compare the actual label by manual and generated labels by proposed algorithm. Table III presents a comparison with actual class ground truth labels and generated labels for each class that matched with provided ground truth labels. There is no previous driving maneuvers data labeling work done. Therefore, direct comparison with previous related work cannot be possible.

\section{B. Challenges}

We analyze the challenges behind the generation of labels, qualitative comparison of manual labeling, and automatic labeling process.

- Human uses self-reasoning without knowing the way of change in time series. For instance, while $a_{x}$ is nonaggressive and $\mathrm{a}_{\mathrm{y}}$ and $\mathrm{w}_{\mathrm{z}}$ are aggressive, according to data change rule it should be LT or RT but the labeler perceived it as aggressive acceleration.

- Human can perceive an event before it occurs. But time series indicates the specific point when the event occurs. By plotting, we find that few event labeling have started 1 or 1.5 seconds earlier it happens and ended 1.5 seconds after the events actually end. Hence, there are many timeseries data that do not indicate the corresponding events by our proposed algorithm.

TABLE II

List OF Class RULE SET

\begin{tabular}{|c|c|}
\hline Rule: & Antecedent $\rightarrow$ Consequent \\
\hline $\mathrm{r}_{1}$ : & $\left(\left(a_{x} \leq 2\right) \wedge\left(a_{x} \geq-2\right)\right) \vee\left(\right.$ eng_a $\left.a_{x} \leq 2\right) \rightarrow$ non_aggressive_ $x$ \\
\hline $\mathrm{r}_{2}$ : & $\left(\left(\mathrm{a}_{\mathrm{y}} \leq 1.5\right) \wedge\left(\mathrm{a}_{\mathrm{y}} \geq-1.5\right)\right) \wedge\left(\mathrm{a}_{\mathrm{y}} \leq 8.5\right) \rightarrow$ non_aggressive_y \\
\hline$r_{3}:$ & $\begin{array}{c}\left(\left(\mathrm{w}_{\mathrm{z}} \leq 0.2\right) \wedge\left(\mathrm{w}_{\mathrm{z}} \geq-0.2\right)\right) \wedge\left(\text { slope } \_\mathrm{w}_{\mathrm{z}} \leq 1.5\right) \rightarrow \\
\text { non_aggressive_z }\end{array}$ \\
\hline $\mathrm{r}_{4}$ : & $\begin{aligned}\left(\mathrm{r}_{1}==\text { False }\right) \wedge & \left(\text { slope_a } \mathrm{a}_{\mathrm{x}}>6\right) \wedge\left(\text { slope_a } \mathrm{a}_{\mathrm{x}} \leq 10\right) \rightarrow \\
& \text { aggressive_acceleration }\end{aligned}$ \\
\hline$r_{5}:$ & $\left(r_{1}==\right.$ False $) \wedge\left(\right.$ slope_a $\left.a_{x}>10\right) \rightarrow$ aggressive_braking \\
\hline $\mathrm{r}_{6}:$ & $\begin{array}{c}\left(\mathrm{a}_{\mathrm{y}}>1.5\right) \vee\left(\left(\text { slope_a } \mathrm{a}_{\mathrm{y}}>8.5\right) \wedge\left(\text { slope_a } \mathrm{a}_{\mathrm{y}}<=10\right)\right) \vee \\
\left(\left(\mathrm{w}_{\mathrm{z}}>0.2\right) \vee\left(\text { slope_( } \mathrm{w}_{\mathrm{z}}>1.5\right)\right) \rightarrow \text { aggressive_RT }\end{array}$ \\
\hline $\mathrm{r}_{7}:$ & $\begin{array}{c}\left(\mathrm{a}_{\mathrm{y}}<-1.5\right) \vee\left(\text { slope_a } \mathrm{a}_{\mathrm{y}}>10\right) \vee\left(\left(\mathrm{w}_{\mathrm{z}}<-0.2\right) \vee\right. \\
\left.\left(\text { slope__} \mathrm{w}_{\mathrm{z}}>1.5\right)\right) \rightarrow \text { aggressive_RT }\end{array}$ \\
\hline
\end{tabular}

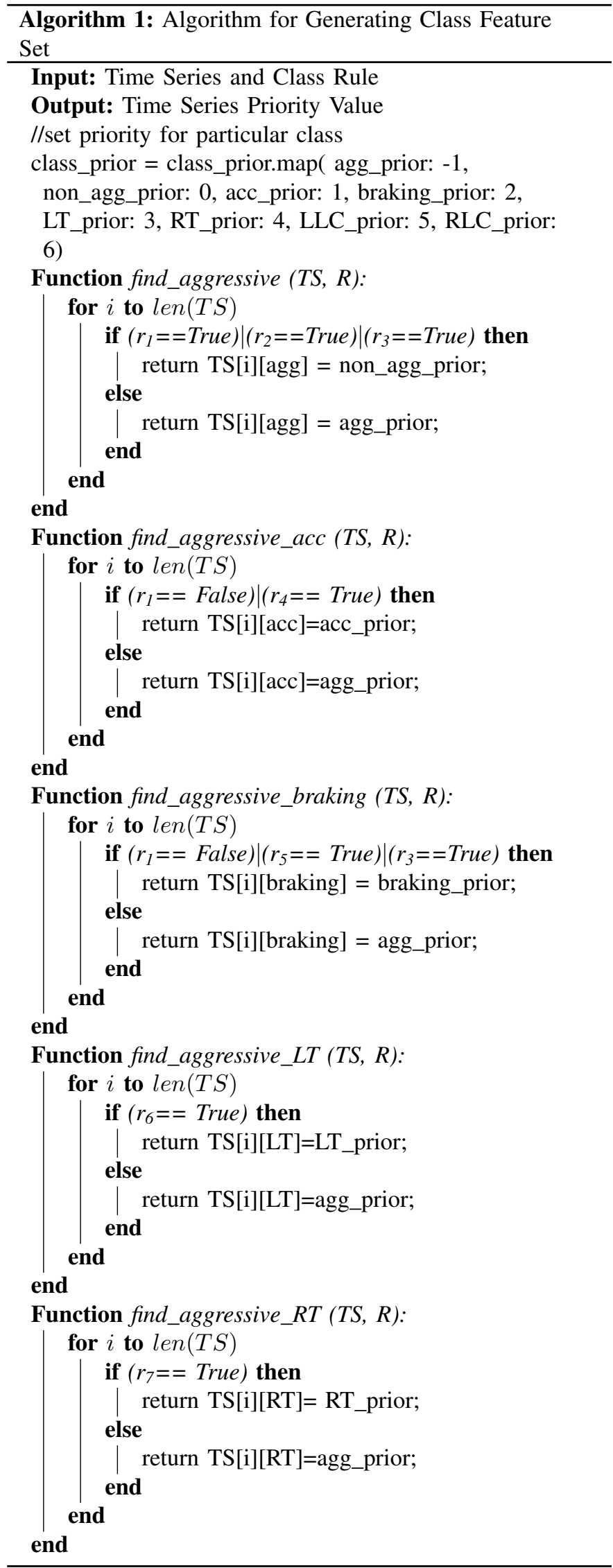



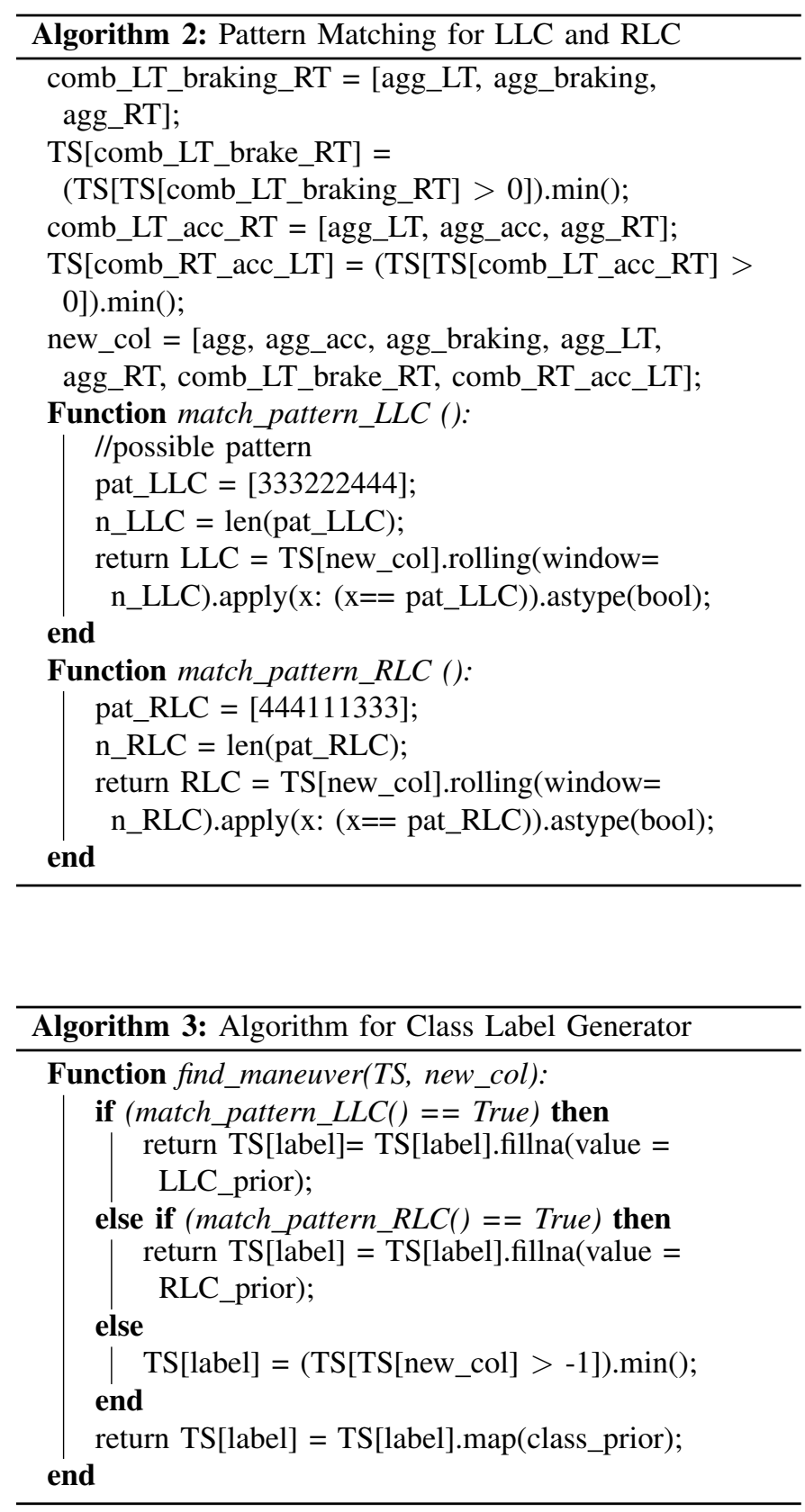

TABLE III

Comparison of Generated Label with Actual Manual Label

\begin{tabular}{|c|c|c|}
\hline Class & $\begin{array}{c}\text { Actual manual } \\
\text { Label }\end{array}$ & $\begin{array}{c}\text { Matched Generated } \\
\text { Label }\end{array}$ \\
\hline Non-aggressive & 2528 & 1804 \\
\hline Acceleration & 2202 & 292 \\
\hline Braking & 1462 & 113 \\
\hline LT & 1946 & 1185 \\
\hline RT & 1948 & 1006 \\
\hline LLC & 427 & 213 \\
\hline RLC & 564 & 282 \\
\hline Total & $\mathbf{1 1 0 7 7}$ & $\mathbf{4 8 9 5}$ \\
\hline
\end{tabular}

- One labeled event contains data patterns of multiple events. For example, before any braking maneuver, there is always a sequence of acceleration and vise-versa but labeled as one event by manual labeling which increases the mismatch of our generated label with manual label.

\section{CONCLUSion}

The paper propose an empirical framework that generates class label of unlabeled time-series driving dataset. For this purpose, we develop a set of class rules based on data change rules of moving vehicle and an algorithm that use the ruleset to generate the class label. We evaluate the performance of proposed algorithm by comparing the generated label and manual label for each class. Moreover, we discuss the challenges related to automatic and manual labeling. If different modality of data such as GPS information, video will be used in such classification, it will need more complex processing and will increase computational complexity. We have a plan to generate a confidence feature along with class feature value. The confidence of an event takes place will depend on the previous similar class priority values.

\section{REFERENCES}

[1] N. Kattukkaran, A. George, T. M. Haridas and S. S. Nasir, "Intelligent accident detection and alert system for emergency medical assistance," in Proc. ICCCI'17, 2017, IEEE, pp. 1-6.

[2] P. Nath and A. Malepati, "IMU based accident detection and intimation system," in Proc. IEMENTech'18, 2018, IEEE, pp.1-4.

[3] R. K. Kodali and S. Sahu, "MQTT based vehicle accident detection and alert system," in Proc. iCATccT'17, 2017, IEEE, pp. 186-189.

[4] S. Sarker, M. S. Rahman and M. N. Sakib, "An approach towards intelligent accident detection, location tracking and notification system," in Proc. ICTP'19, 2019, IEEE, pp. 1-4.

[5] J. Cervantes-Villanueva, D. Carrillo-Zapata, F. Terroso-Saenz, M. Valdes-Vela and A. F. Skarmeta, "Vehicle maneuver detection with accelerometer-based classification," Sensors, vol. 16, no. 10, pp. 1618.

[6] J. Ferreira, E. Carvalho, B. V. Ferreira, C. De Souza, Y. Suhara, A. Pentland and G. Pessin, "Driver behavior profiling: An investigation with different smartphone sensors and machine learning," PLoS one, vol. 12 , no. 4, 2017, pp. e0174959.

[7] E. Carvalho, B. V. Ferreira, J. Ferreira, C. De Souza, H. V. Carvalho, Y Suhara and G. Pessin, "Exploiting the use of recurrent neural networks for driver behavior profiling," in Proc. IJCNN'17, 2017, pp. 3016-3021.

[8] D. Alvarez-Coello, B. Klotz, D. Wilms, S. Fejji, J. M. Gómez and R. Troncy, "Modeling dangerous driving events based on in-vehicle data using Random Forest and Recurrent Neural Network," in Proc. IV'19, 2019, pp. $165-170$.

[9] G. He, Y. Duan, Y. Li, T. Qian, J. He and X. Jia, "Active learning for multivariate time series classification with positive unlabeled data," in Proc. ICTAI' 15, 2015, IEEE, pp. 178-185.

[10] K. Murao and T. Terada, "Labeling method for acceleration data using an execution sequence of activities," in Proc. UbiComp'13, 2013, pp. 611-622.

[11] M. Leodolter, P. Widhalm, C. Plant and N. Brandle, "Semi-supervised segmentation of accelerometer time series for transport mode classification," in Proc. MT-ITS'17, 2017, IEEE, pp. 663-668.

[12] H. L. Cardoso and J. M. Moreira, "Human activity recognition by means of online semi-supervised learning," in Proc. MDM'16, 2016, IEEE, pp. $75-77$.

[13] M. Wu, S. Zhang and Y. Dong, "A novel model-based driving behavior recognition system using motion sensors," Sensors, vol. 16, no. 10, 2016, pp. 1746.

[14] S. Sarker and M. M. Haque, "An approach towards domain knowledge based classification of driving maneuvers with LSTM network," in Proc. IJCACI'20, 2020, Springer, in press.

[15] Driver Behavior Dataset, Sep 4, 2019. Accessed on: Oct 30, 2020 [Online]. Available: https://github.com/jair-jr/driverBehaviorDataset 